\title{
Punción pancreática ecodirigida: estudio multicéntrico
}

\author{
M. C. Garre Sánchez¹, P. Rendón Unceta², A. López Cano ${ }^{3}$, M. Gómez Rubio ${ }^{4}$, B. de Cuenca Morón ${ }^{4}$,
} J. M. Segura Cabral ${ }^{5}$, M. Crespo Sánchez ${ }^{6}$, L. A. Gil Grande 7 , M. J. Varas Lorenzo ${ }^{8}$, J. Just Timoneda9 R. Gómez Rodríguez ${ }^{10}$, J. Galcera Tomás ${ }^{1}$, A. Abraldes Bechiarelli², M. Macías Rodríguez ${ }^{2}$, A. Muñoz Benvenuty $^{3}$, M. Tejada Cabrera ${ }^{3}$, A. Olveira ${ }^{5}$ y E. Palacios Lázaro ${ }^{7}$

Servicios de Aparato Digestivo. ${ }^{1}$ Hospital Virgen de la Arrixaca. Murcia. ${ }^{2}$ Hospital Puerta del Mar. Cádiz. ${ }^{3}$ Clínica Virgen de la Salud. Cádiz. ${ }^{4}$ Hospital Universitario de Getafe. Madrid. ${ }^{5}$ Hospital La Paz. Madrid. ${ }^{6}$ Hospital San Agustín. Avilés, Asturias. ${ }^{7}$ Hospital Ramón y Cajal. Madrid. ${ }^{8}$ Clínica Teknon. Barcelona. ${ }^{9}$ Diagnosis Médica-Cruz Blanca. Barcelona. ${ }^{10}$ Hospital Virgen de la Salud. Toledo

\section{RESUMEN}

Objetivo: en el seno de la Asociación de Ecografía Digestiva se decidió realizar un estudio retrospectivo multicéntrico sobre la punción-aspiración con aguja fina (PAAF) de lesiones ocupantes de espacio pancreáticas, mediante control ecográfico y por vía percutánea, con el objetivo de valorar el rendimiento de dicha técnica y poder compararla con la punción mediante ultrasonografía endoscópica.

Participantes: en el estudio han participado 10 hospitales con 222 pacientes con lesiones pancreáticas entre 8 y $120 \mathrm{~mm}$, sospechosas de malignidad.

Resultados: el análisis de los resultados muestra una sensibilidad del 89\%, especificidad 98\%, valor predictivo positivo 99\% y negativo 74\%, con precisión diagnóstica global 91\%. No encontramos ninguna complicación significativa.

Conclusión: la PAAF de lesiones pancreáticas por vía percutánea es de alta rentabilidad diagnóstica y con pocas y leves complicaciones.

Palabras clave: Punción-aspiración con aguja fina (PAAF). Lesiones ocupantes de espacio pancreáticas. Tumor de páncreas. Punción control ecográfico.

\begin{abstract}
Objective: members of "Asociación de Ecografía Digestiva" decided to carry out a multicenter retrospective study on fine-needle aspiration biopsy for pancreatic space-occupying lesions under ultrasonographic guidance and via the percutaneous route in order to assess this technique's performance versus endoscopic ultrasound-guided biopsy.

Subjects: 10 hospitals for a total of 222 patients with suspiciously malignant, 8-120-mm pancreatic lesions were included in the study.

Results: the analysis of results shows a sensitivity of $89 \%$, a specificity of $98 \%$, a positive predictive value of $99 \%$, and a negative predictive value of $74 \%$, for an overall diagnostic accuracy of 91\%. No major complications occurred.

Conclusion: percutaneous fine-needle aspiration for pancreatic lesions is highly cost-effective and has few and mild complications.
\end{abstract}

Key words: Fine-needle aspiration. Pancreatic space-occupying lesions. Pancreatic tumor. Ultrasound guided biopsy.

Garre Sánchez MC, Rendón Unceta P, López Cano A, Gómez Rubio M, De Cuenca Morón B, Segura Cabral JM, Crespo Sánchez M, Gil Grande LA, Varas Lorenzo MJ, Just Timoneda J, Gómez Rodríguez R, Galcera Tomás J, Abraldes Bechiarelli A, Macias Rodríguez M, Muñoz Benvenuty A, Tejada Cabrera M, Olveira A y Palacios Lázaro E. Punción pancreática ecodirigida: estudio multicéntrico. Rev Esp Enferm Dig 2007; 99: 520-524.

Recibido: 11-03-07.

Aceptado: 09-07-07.

Correspondencia: Carmen Garre Sánchez. $\mathrm{P}^{\circ}$. Escultor J. Gonzalez Moreno, $4,3^{\circ}$ C. 30002 Murcia.e-mail: c.garre@ono.com

\section{INTRODUCCIÓN}

Los primeros trabajos de punción-aspiración con aguja fina (PAAF) de órganos abdominales con control ecográfico los publicaron Rasmussen y Holm en 1972 (1), sin embargo esta técnica alcanza gran auge una década más tarde, debido a la aparición de equipos de tiempo real, 
que determinó un gran desarrollo de la misma, llegando en pocos años a realizarse PAAF en todos los órganos de la cavidad abdominal $(2,3)$. El reciente desarrollo de la ultrasonografía endoscópica (USE) y la PAAF de lesiones pancreáticas mediante USE, nos plantea si en un futuro la PAAF percutánea podrá ser desplazada por la PAAF con USE o ambas serán complementarias. En el seno de la Asociación de Ecografía Digestiva (AED), surge esta inquietud y se decide realizar un estudio retrospectivo de las PAAF percutáneas realizadas en los distintos Servicios de Aparato Digestivo que realizan intervencionismo ecográfico.

\section{PACIENTES, MATERIAL Y MÉTODO}

Se solicitó a las Unidades de Ecografía de los Servicios de Aparato Digestivo que revisaran de forma retrospectiva las punciones percutáneas pancreáticas mediante control ecográfico. Se elaboró y remitió por correo electrónico una hoja de recogida de datos y una base de datos en Excel $^{\circledR}$, para utilizar una de ellas indistintamente. Se aportaron pacientes desde los Servicios de Aparato Digestivo de los Hospitales Puerta del Mar (Cádiz), Virgen de la Arrixaca (Murcia), Clínica Virgen de la Salud (Cádiz), Universitario de Getafe (Madrid), La Paz (Madrid), San Agustín (Avilés), Ramón y Cajal (Madrid), Clínica Teknon (Barcelona), Diagnosis Médica-Cruz Blanca (Barcelona), Virgen de la Salud (Toledo). La recogida y análisis estadístico de los datos aportados se realizó con el programa SPSS 12.0 para Windows.

En todos los casos la PAAF se realizó con control ecográfico directo en tiempo real, se utilizaron agujas de $22 \mathrm{G}$ en el $80 \%$ de los casos, en el resto de $25 \mathrm{G}$ y $20 \mathrm{G}$ (Figs. 1 y 2). Los pacientes estaban en ayunas, con plaquetas y coagulación superiores a 50.000 y $50 \%$ de actividad de protrombina. El patólogo estaba en la sala de ecografía durante la punción en 157 (71\%) de las PAAF, donde realizó una tinción rápida para un primer estudio de la muestra, no se hizo control en 65 (29\%). En 6 hospitales de los 10 que participan en el estudio, el patólogo habitualmente está presente en la PAAF.

La confirmación del diagnóstico se realizó mediante cirugía, necropsia, otras pruebas de imagen y/o evolución clínica de los pacientes.

\section{RESULTADOS}

Se revisaron de forma retrospectiva 222 PAAF realizadas entre los años 1986 y 2005, en 222 pacientes; $119(54 \%)$ varones y $103(46 \%)$ mujeres de edades comprendidas entre 27 y 93 años con una edad media de $63 \pm 12$ años. La edad media de los varones fue menor que la de las mujeres $60 \pm 12 v s .65 \pm 12$ años respectivamente, $\mathrm{p}=0,006$.
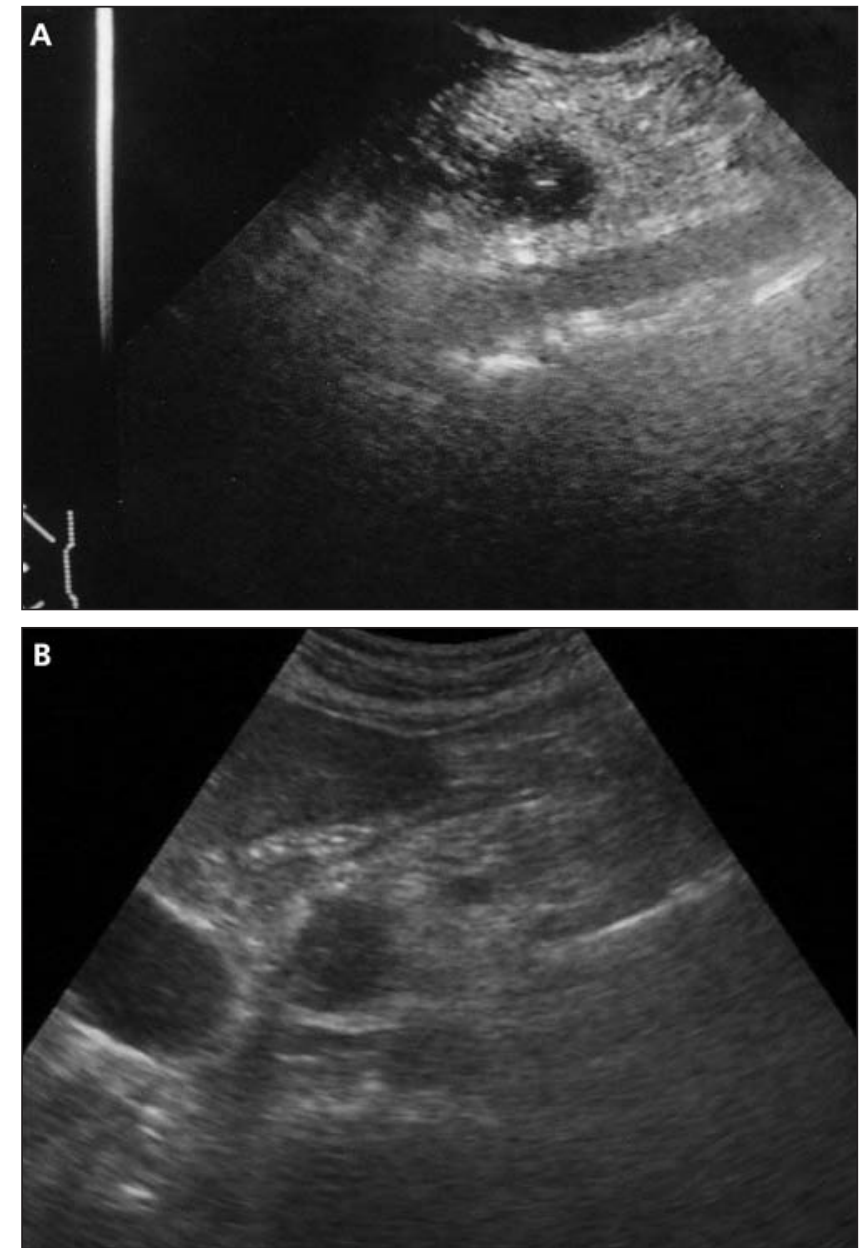

Fig. 1. A. Neoplasia de cabeza de páncreas en corte longitudinal, se observa la punta de la aguja en el centro de la lesión. B. Neoplasia cabeza de páncreas en corte transversal.

Los hábitos tóxicos, antecedentes y datos clínicos conocidos se exponen en la tabla I. El principal síntoma de inicio fue el dolor abdominal, en más de la mitad de los casos, seguido de ictericia en 32\%, ambos síntomas estaban presentes en el 18\% de los casos, y el $63 \%$ de los pacientes con ictericia también tenían dolor.

Tabla I. Antecedentes y clínica por la que consultan los pacientes

\begin{tabular}{lrc}
\hline & $N^{\circ}$ pacientes & $\begin{array}{c}N^{0} \text { total pacientes } \\
\text { con dato conocido }\end{array}$ \\
\hline Bebedor & $76(64 \%)$ & 118 \\
Fumador & $38(32 \%)$ & 117 \\
Bebedor + fumador & $27(24 \%)$ & 114 \\
Patología biliar & $33(20 \%)$ & 166 \\
Dolor abdominal & $118(57 \%)$ & 157 \\
Ictericia & $52(32 \%)$ & 161 \\
Dolor + ictericia & $27(18 \%)$ & 152 \\
\hline
\end{tabular}



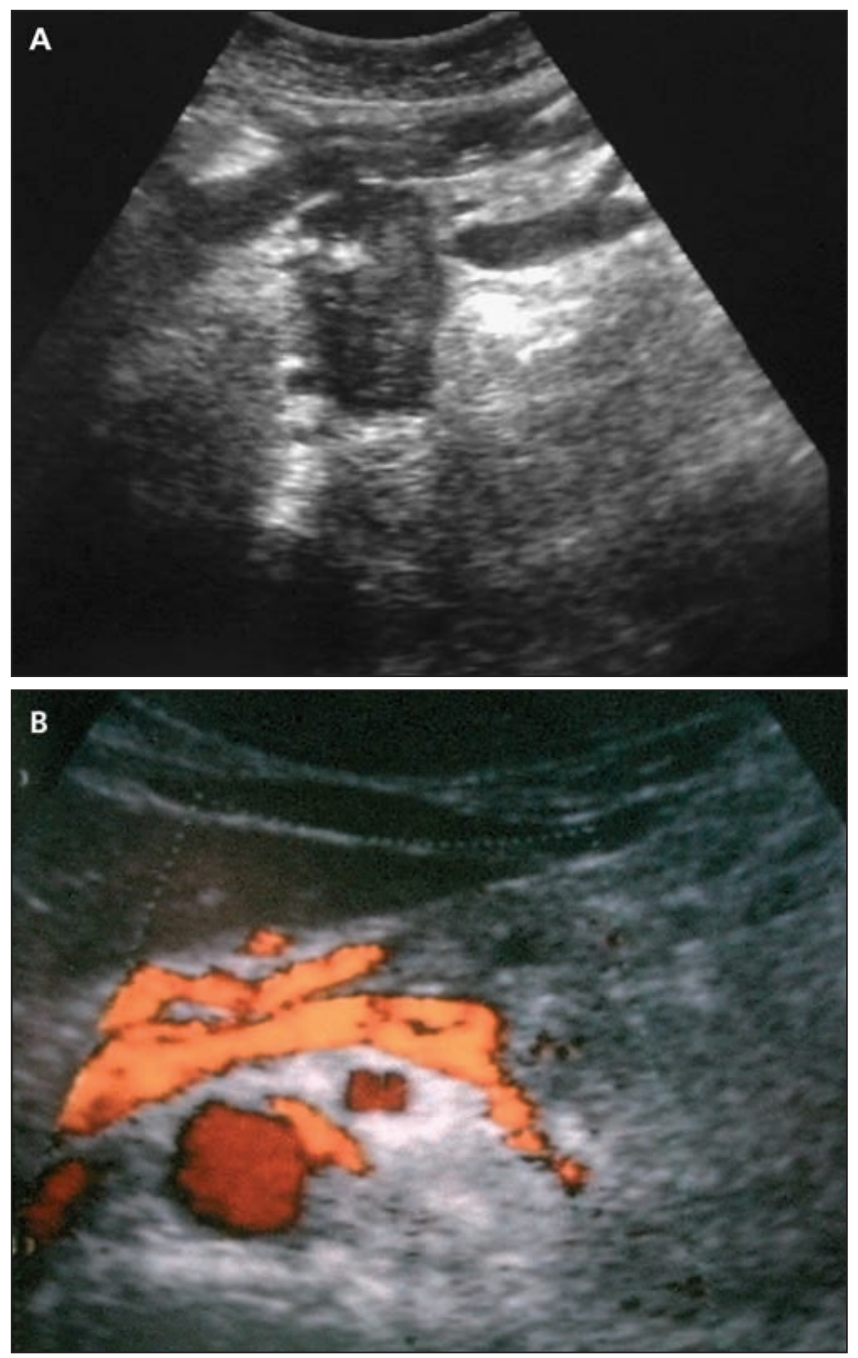

Fig. 2. A. Neoplasia de cabeza de páncreas de gran tamaño. B. Neoplasia de cola pancreática con invasión vascular.

Los hallazgos ecográficos asociados a la presencia de la lesión ocupante de espacio (LOE) se reflejan en la tabla II. El colédoco estaba dilatado en $65(35 \%)$ y el Wirsung en 30 (14\%) de los 185 y 209 pacientes respectivamente en los que se conocía el dato. La presencia de adenopatías retroperitoneales se observó en el $25 \%$ de los pacientes y metástasis hepáticas en menor proporción, pues en general cuando las encontramos, se realiza la PAAF sobre ellas y sólo se hace sobre el páncreas cuando son muy pequeñas; la citología no es concluyente o hay duda de si dichas metástasis son de origen pancreático.

El tamaño de las LOES osciló entre 8 y $120 \mathrm{~mm}$, con un tamaño medio de 37,5 $\pm 16 \mathrm{~mm}$. La localización más frecuente fue en la cabeza pancreática 146 (67\%), 50 (23\%) en el cuerpo y $21(10 \%)$ en la cola. La imagen ecográfica mostró que $163(77 \%)$ eran hipoecogénicas, 31 (15\%) heterogéneas, $11(5 \%)$ isoecogénicas y $6(3 \%)$ hiperecogénicas. La consistencia fue sólida en 164 (79\%), líquida en 22 $(10 \%)$ y $23(11 \%)$ de las lesiones (Tabla III).
Tabla II. Hallazgos ecográficos

\begin{tabular}{lcc}
\hline & $N^{\circ}$ pacientes & $\begin{array}{c}N^{\circ} \text { pacientes } \\
\text { dato conocido }\end{array}$ \\
\hline Dilatación colédoco & $65(32 \%)$ & 185 \\
Dilatación Wirsung & $30(14 \%)$ & 209 \\
Signos PC & $20(12 \%)$ & 173 \\
Adenopatías retroperitoneales & $43(25 \%)$ & 170 \\
Metástasis hepáticas & $33(16 \%)$ & 209 \\
Otras metástasis & $15(9 \%)$ & 168 \\
\hline
\end{tabular}

Tabla III. Características ecográficas de las LOES pancreáticas

\begin{tabular}{lc}
\hline Tamaño $(\mathrm{mm}): \begin{array}{c}\mathrm{x} \pm \mathrm{SD} \\
\text { Rango }\end{array}$ & $37 \pm 16$ \\
& $8-120$ \\
Ecogenicidad & \\
Hipo & $163(67 \%)$ \\
Hetero & $31(15 \%)$ \\
Iso & $11(5 \%)$ \\
Hiper & $6(3 \%)$ \\
Consistencia & \\
Sólida & \\
Líquida & $164(79 \%)$ \\
Mixta & $22(10 \%)$ \\
\hline
\end{tabular}

Se obtuvo material suficiente para el estudio citológico en 202 (91\%) de los pacientes, no se consiguió en 20 (9\%). Los resultados de las 222 PAAF se reflejan en la tabla IV.

Tabla IV. Resultados 222 PAAF pancreáticas

\begin{tabular}{lc}
\hline Verdaderos positivos (VP) & $134(60 \%)$ \\
Verdaderos negativos (VN) & $50(22 \%)$ \\
Falsos positivos (FP) & $1(0,5 \%)$ \\
Falsos negativos (FN) & $17(8 \%)$ \\
Material insuficiente (MI) & $20(9 \%)$ \\
\hline
\end{tabular}

El único falso positivo comunicado en esta serie, ocurrió en el año 1993, con poca experiencia aún el la técnica, tanto por los ecografistas como por los patólogos; el estudio citológico lo clasificó como carcinoma cuando en realidad el diagnóstico final fue una pancreatitis crónica.

Se ha realizado PAAF sobre 22 LOES de consistencia líquida (dato conocido en 209 casos), de ellas 2 (10\%) eran malignas.

Con estos resultados se deduce que la técnica ha tenido una precisión diagnóstica global del 91\%, sensibilidad del $89 \%$, especificidad del $98 \%$, valor predictivo de los resultados positivos del $99 \%$ y negativos del 74\% (Tabla V). 
Tabla V. Resultados PAAF pancreáticas

\begin{tabular}{lcccc}
\hline Sensibilidad & Especificidad & VP+ & VP- & Precisión diag. \\
\hline $89 \%$ & $98 \%$ & $99 \%$ & $74 \%$ & $91 \%$ \\
\hline
\end{tabular}

En nuestra serie de las 222 LOES, 156(73\%) fueron $\geq 3 \mathrm{~cm}$ y $59<3 \mathrm{~cm}$. La comparación de los dos grupos demostró que en el de menor tamaño hubo más proporción de casos con material insuficiente para el diagnóstico, 7 (12\%) vs. 13 (8\%), si bien esta diferencia no fue estadísticamente significativa. Tampoco encontramos diferencias entre benignidad y malignidad en relación al tamaño de las lesiones (Tabla VI).

$\mathrm{Al}$ analizar los 17 falsos negativos observamos que 3 (18\%) LOES eran menores de $3 \mathrm{~cm}$, mientras que 14 (82\%) eran mayores de $3 \mathrm{~cm}$, no hemos encontrado diferencias estadísticamente significativas entre ambos grupos.

Tabla VI. Relación entre tamaño de las lesiones y citología

\begin{tabular}{lccccc}
\hline & & \multicolumn{3}{c}{ Citología } & \multirow{2}{*}{ Total } \\
\cline { 3 - 4 } & & No material & Maligno & Benigno & \\
\hline Tamaño & $<3 \mathrm{~cm}$ & $7(12 \%)$ & $31(52 \%)$ & $21(36 \%)$ & 59 \\
& $>3 \mathrm{~cm}$ & $13(8 \%)$ & $99(64 \%)$ & $44(28 \%)$ & 156 \\
Total & & $20(9 \%)$ & $130(61 \%)$ & $65(30 \%)$ & 215
\end{tabular}

* Las comparaciones estadísticas se limitaron a los datos con obtención adecuada de muestra.

El 74\% de las lesiones sólidas fueron malignas, frente al $11 \%$ de las líquidas y 59\% de las mixtas, estas diferencias son estadísticamente significativas.

La tolerancia fue muy buena en todos los pacientes, no se ha comunicado ninguna complicación significativa.

\section{DISCUSIÓN}

La ecografía abdominal es la técnica de primera elección en el estudio de los pacientes con sospecha de tumor pancreático. Cuando se seleccionan los pacientes con exploración satisfactoria del área pancreática, la ecografía muestra una sensibilidad del $70-94 \%$ para la detección del tumor (4). La especificidad de la ecografía en el diagnóstico del cáncer de páncreas es muy variable en los diferentes estudios, pero se puede considerar baja en las LOES que aparecen en el contexto de una pancreatitis aguda o crónica; de hecho, hasta el $50 \%$ de las masas sometidas a PAAF pueden ser inflamatorias y la precisión diagnóstica de la ecografía para distinguir entre los distintos tipos tumorales oscila entre el 35 y $66 \%(4,5)$. En nuestra serie un $22 \%$ de los casos fueron lesiones benignas y en el $12 \%$ de los pacientes se encontraron signos ecográficos de pancreatitis crónica. En el momento actual la principal indicación de la PAAF pancreática es la dis- tinción entre procesos inflamatorios (pancreatitis aguda focal, pancreatitis crónica) y sus secuelas (pseudoquistes y abscesos) de las neoplasias (6).

La PAAF de las LOES pancreáticas con ecografía convencional ha demostrado alta sensibilidad, especificidad y precisión diagnóstica en las distintas series publicadas (Tabla VII).

Tabla VII. Sensibilidad y precisión diagnóstica en PAAF pancreáticas en algunos estudios

\begin{tabular}{lcccc}
\hline Autores (año) & $N^{\circ}$ casos & $\begin{array}{c}\text { Sensibilidad } \\
\%\end{array}$ & $\begin{array}{c}\text { Especificidad } \\
\%\end{array}$ & $\begin{array}{c}\text { Precisión } \\
\text { diagnóstica } \\
\text { global \% }\end{array}$ \\
\hline Ayuso (1985) (7) & 27 & 88 & 100 & 70 \\
Hancke (1985) & 203 & 71 & 100 & - \\
Hall-Craggs (1986) (8) & 240 & 71 & 100 & 76 \\
Segura Cabral (1989) (2) & 14 & 92 & 100 & 90 \\
Fornari (1992) (9) & 59 & 74 & 100 & 89 \\
Sperti (1994)(10) & 83 & 91 & 100 & 93 \\
Di Stasi (1998) (11) & 510 & 87 & 100 & 91 \\
Garre (1999) (12) & 38 & 85 & 100 & 88 \\
\hline
\end{tabular}

En nuestra serie no obtuvimos material suficiente para el diagnóstico en 20 pacientes (9\%), similar al estudio multicéntrico italiano de 572 pacientes que no se obtuvo material en $62(10,8 \%)$ (11). Los falsos negativos fueron $17(8 \%)$ en nuestra serie. Las razones de la no presencia de material o falsos negativos en los tumores pancreáticos son:

- Presencia de fibrosis y áreas de necrosis alrededor y en el interior del tumor.

- Pequeño tamaño de la lesión diana.

- Dificultad del citólogo para diagnosticar los tumores muy bien diferenciados.

Di Stasi y cols. (11) no encuentran falsos negativos en los casos de metástasis pancreáticas, linfomas, abscesos o páncreas normal con pseudomasa. En los pseudoquistes. adenocarcinoma y neoplasias quísticas observan que la capacidad diagnóstica de la PAAF es 97,86 y $62 \%$, respectivamente, mientras que es muy inferior en las pancreatitis con masa y tumor neuroendocrino: 35 y $33 \%$ respectivamente.

La presencia de falsos positivos es excepcional y sitúan esta técnica con un valor predictivo positivo de los más altos de los diferentes órganos (6), incluso algunos estudios comparativos entre PAAF con aguja fina y biopsia con aguja gruesa muestran mayor obtención de material suficiente para el diagnóstico con aguja fina $97 v s .89 \%$ y mejor precisión diagnóstica de la citología frente a la histología 78 vs. $46 \%$ (13). En los estudios más amplios se obtienen similares resultados con citología o microhistología (14). Estudios recientes que comparan citología e histología obtenidas mediante USE muestran una tendencia a mayor sensibilidad y precisión diagnóstica de la histología, aunque no estadísticamente significativas (15). 
La PAAF para estudio citológico no sólo permite conocer la naturaleza maligna del proceso, sino que también permite establecer el diagnóstico histológico, incluso en tumores muy poco frecuentes como el carcinoma adenoescamoso o los linfomas $(16,17)$.

El $10 \%$ de los quistes pancreáticos son tumorales. En nuestra serie también el $11 \%$ de las lesiones quísticas fueron malignas. Hasta un $30 \%$ de los tumores quísticos pancreáticos son inicialmente tratados como pseudoquistes (18).

La ultrasonografía endoscópica (USE) permite visualizar y realizar PAAF sobre LOES de menor tamaño; tiene gran valor en el estadificación del tumor que comprende localización, invasión de órganos vecinos como pared duodenal o estructuras vasculares, existencia de circulación colateral y adenopatías paratumorales o metastásicas (19).

Las complicaciones de la PAAF con control ecográfico son pocas y generalmente leves. La mortalidad con aguja fina es de 0,008 a $0,03 \%$, y las causas más frecuentes son hemorragias, pancreatitis necrótico-hemorrágicas y shock séptico $(20,21)$. En nuestra serie no se presentaron complicaciones significativas.

En conclusión, la PAAF de lesiones pancreáticas con ecografía tiene alta rentabilidad diagnóstica y sigue siendo de gran utilidad en el momento actual, es sencilla de realizar y poco molesta para el paciente. La PAAF mediante USE supone un importante avance pues es capaz de llegar a lesiones de pequeño tamaño inaccesibles a la ecografía convencional y completar la estadificación del tumor, sin embargo es más compleja y precisa sedación profunda con anestesista. En el momento actual pensamos que ambas técnicas son complementarias, la PAAF percutánea no precisa anestesia, es menos molesta y con menos riesgos que la USE; por otra parte en los centros en los que no se dispone de USE el traslado del paciente puede demorar el diagnóstico. En el futuro, como en el presente, ambas técnicas coexistirán y cuando sea necesaria una PAAF de una lesión pancreática la vía de acceso se decidirá en base a las características de la lesión, del paciente y del centro hospitalario.

\section{BIBLIOGRAFÍA}

1. Rasmussen SN, Holm HH, Kristensen JK, Barlebo H. Ultrasonically guided liver biopsy. Br Med J 1972; 2: 500-2.

2. Segura Cabral JM, Mora Sanz P, Villeta Caraballo E. Punción-aspiración diagnóstica guiada por ultrasonidos. En: JM Segura Cabral.
Ecografía en patología digestiva. Barcelona: Ed. Doyma; 1990. p. 137-44.

3. Capdevilla A, Varas MJ, Sentis M. Punción espirativa con aguja fina guiada o bajo control continuo con ultasonidos en el diagnóstico y tratamiento de la patología digestiva. Rev Esp Enferm Dig 1985; 68 (4): 331-6.

4. Macías Rodríguez MA, Rendón Unceta P, Martín Herrera L. Ecografía convencional en el cáncer de páncreas. Ecodigest 2004; 6 (2): 54 6.

5. Gil Grande LA, Miquel Plaza J, García-Hoz F, Cuesta Roca C, García González M, López San Román A, et al. Rentabilidad de la punción-aspiración con aguja fina con control ecográfico en el diagnóstico de patología ocupacional intraabdominal. Ecodigest 2000; 2 (3): 125-31

6. López Cano A, Muñoz Benvenuty A, Herrera MD. Ecografía intervencionista. En: José María Segura. Ecografia abdominal. Madrid: Ed. Norma; 1996. p. 457-503.

7. Ayuso MC, Bru C, Faus R, Bruguera M, Rodés J. La punción aspirativa con aguja fina (PAAF) bajo control ecográfico en el diagnóstico de tumores hepáticos, pancreáticos y retroperitoneales. Revisión de 176 casos. Gastroenterol Hepatol 1985; 8 (1): 18-23.

8. Hall-Craggs MA, Lees WR. Fine-needle aspiration biopsy: pancreatic and biliary tumors. AJR 1986; 147: 399-403.

9. Fornari F, Buscarini L Ultrasonically-guided fine-needle biopsy of gastrointestinal organs: indications, results and complications. Dig Dis 1992; 10 (3): 121-33.

10. Sperti C, Pasquali C, Di Prima F, Rugge M, Petrin P, Costantino V, et al. Percutaneous CT-guided fine needle aspiration cytology in the differential diagnosis of pancreatic lesions. Ital J Gastroenterol 1994; 26 (3): 126-31.

11. Di Stasi M, Lencioni R, Solmi L, et al. Ultrasound-guided fine needle biopsy of pancreatic masses: results of a multicenter study. AJG 1998; 93 (8): 1329-33.

12. Garre C, Sola J, Bas A, Guillén B, Campos M. Punción aspiración con aguja fina con control ecográfico de lesiones abdominales. Ecodigest 2000; 2 (4): 183-7.

13. Wittemberg J, Mueller PR, Ferrucci JT, et al. Percutaneous core biopsy of abdominal tumors using 226 needles. Futher observations. AJR 1982; 139: 75-80.

14. Lencioni R, Balognesi P, Cilotti A, et al. Ultrasound-guided fine needle biopsy of the pancreas: Smear cytology versus microhistology. Eur Radiol 1992; 2: 252-7.

15. Iglesias-García J, Domínguez-Muñoz E, Lozano-León A, et al. Impact of endoscopio ultrasound-guided fine leedle biopsy for diagnosis of pancreatic masses. Worl J gastroenterol 2007; 13 (2): 289-93.

16. Erdozain Sosa JC, Presa Valle M, González Murillo M, Muñoz Núñez F, Lizasoain Urkola J, Segura Cabral. Carcinoma adenoescamoso de páncreas: diagnóstico por punción citológica percutánea bajo control ecográfico. Rev Esp Enferm Digest 1993; 84 (2): 130-2.

17. Arcari A, Anselmo E, Bernuzzi P, et al. Primary pancreatic lymphoma. Reporto f five cases. Haematologiaca 2005; 90 (2): ECR09.

18. Rendón Unceta P, Macías Rodríguez A, Guillén Mariscal P, Martín Herrera L. Ecografía intervencionista terapéutica en la patología pancreática. Ecodigest 1999; 1 (4): 107-13.

19. Maluenda Colomer. Ecoendoscopia biliopancreática. En: Just Timoneda J. Avances en ecografía digestiva. Barcelona: Ed. Degsoc Médica; 2004. p. 125-48.

20. Edward H, Smith MD. Complications of percutaneous abdominal fine-needle biopsy. Radiology 1991; 178: 253-8.

21. Livraghi T, Damascelli B, Lobardi C, Spangoli I. Risk in fine-needle abdominal biopsy. J Clin Ultrasound 1983; 11: 77-81. 